\title{
Des personnalités évitantes plus évitantes que d'autres : intérêt du test de Rorschach dans la compréhension des facteurs qui s'opposent au processus psychothérapique
}

\section{Several avoidant personalities are more avoidant than others: The interest of the Rorschach test in objectifying some factors opposing psychotherapy}

\author{
Dana Castro $^{\mathrm{a}, *}$, Damien Fouques ${ }^{\mathrm{b}}$ \\ ${ }^{a}$ Laboratoire CLIPSYD, EA 4430, 186, boulevard Haussmann, 75008 Paris, France \\ ${ }^{\mathrm{b}}$ Psychologie clinique, laboratoire CLIPSYD, EA 4430, UFR SPSE, université Paris, Nanterre, France
}

\author{
Mots clés : \\ Enquête rétrospective \\ Estime de soi \\ Narcissisme \\ Personnalité évitante \\ Prévalence \\ Psychothérapie \\ Test de Rorschach \\ Trouble de la personnalité
}

\section{RÉ S U M É}

Les troubles évitants de la personnalité (TEP) représentent un motif fréquent de consultation psychologique ou psychiatrique. La grande majorité des patients consultant pour des troubles évitants de la personnalité décrivent une importante souffrance psychique ainsi qu'une faible qualité de vie principalement, du fait de leur isolement. Lorsqu'ils s'engagent dans une psychothérapie, et indépendamment du modèle thérapeutique proposé, certains patients retirent un réel bénéfice de leur traitement, alors que d'autres arrêtent le suivi prématurément, généralement après les premiers signes d'amélioration. L'objectif de cette étude est d'investiguer, à partir du test de Rorschach, les variables qui pourraient rendre compte des différences existant dans la réponse au traitement psychothérapique des patients présentant un trouble évitant de la personnalité.

Matériel et méthodes. - Les protocoles Rorschach (Système Intégré) de 20 patients présentant une pathologie évitante de la personnalité, diagnostiquée selon les critères du DSM IV-TR par un psychiatre référent, ont été inclus dans une étude rétrospective et divisés en deux sous-groupes : le premier AT ( $n=11)$ comprenait des patients ayant achevé leur psychothérapie (intégrative) d'un commun accord avec leur psychothérapeute ; le deuxième, NAT $(n=9)$ a été constitué de patients ayant prématurément interrompu leur psychothérapie après une amélioration comportementale significative, mais subjective. Le recueil des données a été effectué par l'intermédiaire du test de Rorschach, proposé en début de prise en charge. Les protocoles de l'étude ont été cotés deux fois : une première, préalablement au début de la psychothérapie et une deuxième par les deux auteurs lors de l'inclusion dans l'étude. Les données ont été analysées par des statistiques descriptives et par des tests de Mann Withney et de Fisher.

Résultats. - Les résultats ont montré qu'une majorité des patients terminant prématurément leur psychothérapie présentent (1) des traits narcissiques de personnalité ( $\mathrm{Fr}+\mathrm{rF}>\mathrm{N}$ ) (définis au Rorschach comme une attitude de sur-évaluation de la valeur personnelle s'accompagnant de mécanismes de défense tels que l'idéalisation, l'externalisation et la rationalisation) ; (2) une importante détresse émotionnelle et (3) une rigidité cognitive.

Discussion. - Si dans les premières phases de la psychothérapie, la souffrance psychique représente un des leviers de changement, la présence des traits narcissiques, de la détresse et de la rigidité cognitive interfère négativement avec l'adhésion au traitement. Le statut psychologique de ces variables est discuté dans la perspective d'un conflit approche/évitement et dans celle d'un processus défensif permettant au sujet d'éviter ses véritables préoccupations ou la pleine confrontation à un état affectif marqué par la honte ou/et la culpabilité. 
Keywords:

Avoidant personality disorder

Narcissism

Prevalence

Personality trouble

Prévalence

Psychotherapy

Retrospective survey

Rorschach test

Self-esteem
Avoidant personality disorders are (APD) among the most prevalent personality disorders and a frequent request for consultations. Patients suffering from this disorder describe high levels of distress and low quality of life. Some of the consulting patients benefit from a genuine improvement in psychotherapy while others drop out after the first signs of recovery. The aim of this study is to investigate, from the Rorschach Test, the variables accounting for the treatment response differences in a population of APD. Methods. - Twenty Rorschach protocols collected from 20 APD patients, diagnosed as such according to the DSM-IV-TR by their psychiatrist, have been included in a retrospective study and subdivided in 2 groups. The first, AT ( $n=11)$, had been composed by patients that completed their psychotherapy (integrative) in agreement with their psychotherapist recommendations, and the second, NAT $(n=9)$ by drop-outs who interrupted their treatment in spite of their psychotherapist recommendations, after a subjective feeling of improvement. The research material has been collected through the Rorschach test (Comprehensive System); the protocols had been scored twice : prior to the therapeutic process and prior to inclusion in the study. Data had been analyzed with descriptive statistics as well as with the Mann Withney and Fisher's exact test.

Results. - Results had shown that the patients that drop out prematurely from therapy present, in the Rorschach test (1) narcissistic traits $(\mathrm{Fr}+\mathrm{rF}>\mathrm{N})$, which are defined as a tendency to overestimate his own value through defense mechanisms such as idealization, externalization and denial; (2) anxiety and chronic stress and (3) cognitive rigidity.

Discussion. - If in the early stages of psychotherapy the high level of distress acts as a stout lever for help seeking, when improvement arise, narcissistic traits, chronic distress and rigidity become impediments to treatment adherence. The psychological status of these variables is discussed in the light of the approach/avoidance theory where they act as a coping emotion-focused strategy aiming to prevent the patient from facing guilt, shame or more anxiety stemming from the psychotherapy deepening.

\section{Introduction}

La personnalité évitante représente un trouble fréquent de personnalité, invalidant et sévère, avec une prévalence estimée à approximativement $2 \%$ de la population générale [8,33,37,39,40] et à 10 à $20 \%$ des patients consultant en psychiatrie [21,32]. Les dysfonctionnements de ces personnalités s'observent au niveau de l'estime de soi, de l'identité et des relations interpersonnelles $[13,41]$. L'estime de soi, très basse chez ces personnalités, est ancrée dans la peur du jugement et de la moquerie (gelotophobia) [17]. Elle est maintenue par une très importante centration sur soi qui entrave la capacité à comprendre ses propres états mentaux comme ceux d'autrui [27].

L'identité est construite sur des bases irréalistes, autour d'un sentiment inconditionnel d'inadéquation et d'infériorité qui véhicule un ressenti d'inconsistance et pousse le sujet à chercher " le sens de soi-même " (sense of self) [30,34]. L'inconsistance identitaire est renforcée, au niveau cognitif, par une mémoire biographique insuffisante, car trop généralisante, qui empêche la description détaillée et conscientisée de l'expérience [12].

Les relations interpersonnelles sont marquées par une grave problématique d'attachement où l'anxiété d'abandon joue un rôle central ainsi que l'anticipation d'une frustration intolérable [14]. Le dysfonctionnement relationnel est maintenu par une dérégulation émotionnelle où les émotions (négatives comme positives) et les sensations corporelles sont perçues comme menaçantes pour l'identité et donc redoutées [34].

Pour ces raisons, les principales stratégies de coping de ces personnalités sont essentiellement basées sur l'évitement de toute situation jugée potentiellement dangereuse pour l'estime de soi et/ou la recherche démesurée de réassurance et de validation.

L'évitement se manifeste soit directement par l'isolement social : " mieux vaut être seul pour le meilleur et pour le pire " [34], soit par la consommation de substances psychoactives diverses, l'alcool par exemple [3].

D'un point de vue nosographique, le trouble de la personnalité évitante est très proche de la phobie sociale et est considéré par nombre d'auteurs comme une forme plus diversifiée, au niveau symptomatique, et plus intense au niveau émotionnel que celle-ci $[13,15]$.

Le traitement psychologique de ces personnalités est complexe et vise la réduction symptomatique, par l'acquisition d'une plus grande flexibilité cognitive et une meilleure régulation émotionnelle. Selon les modèles thérapeutiques, les cibles thérapeutiques seront :

- dans le modèle cognitivo-comportemental, la réfutation des croyances négatives par une observation active des situations sociales, l'exposition, la restructuration cognitive et la reconstruction de l'image de soi [30] ;

- dans le modèle psychanalytique, la restauration de la relation d'objet et la diversification de l'éventail défensif [28,29].

Toutefois, quels que soient le modèle théorique ou les techniques de prise en charge de ces personnalités, l'installation d'une alliance thérapeutique contenante et authentique est une étape indispensable à l'efficacité thérapeutique [23].

Or, pour construire l'alliance thérapeutique, certains auteurs préconisent le passage par une évaluation psychologique approfondie, préalablement à l'engagement dans la psychothérapie $[19,20]$. En effet, ces auteurs montrent que l'administration d'un examen psychologique au moyen de tests et surtout la restitution des résultats au sujet contribuent significativement au développement positif de l'alliance thérapeutique [23].

C'est dans le cadre d'évaluations psychologiques préalables au traitement psychothérapique avec des personnalités évitantes que s'inscrit ce travail. Les auteurs ont l'habitude de proposer à leurs patients, avant la thérapie, une évaluation projective (dont le test de Rorschach en Système Intégré) dans un double objectif :

- d'une part, ils tentent ainsi d'approcher la singularité psychologique de leur patient (traits de personnalité inattendus, mécanismes de défense, niveaux spécifiques d'organisation de la personnalité, ressources disponibles...) ce qui contribue à une compréhension fine et globale du patient, de ses besoins et à la définition d'un " plan de traitement " sur mesure [5, p. 436] ; 
- d'autre part, ils visent, par la restitution des résultats, l'établissement d'une alliance thérapeutique positive pour un futur travail psychothérapeutique fructueux.

Dans leur pratique avec des sujets porteurs de troubles évitants de la personnalité, les auteurs ont souvent observé, au test de Rorschach, une configuration assez insolite. Il s'agissait de l'association des critères sémiologiques attendus des personnalités évitantes ${ }^{1}$ et conformes au DSM, à la présence inattendue de traits narcissiques confirmés ${ }^{2}$ (grandiosité du soi et externalisation des conflits) chez certains patients.

Cette configuration psychologique et plus particulièrement la sur-valorisation de soi paraissaient quelque peu surprenantes chez des sujets dont les principales caractéristiques sont, au contraire, la faible estime de soi et l'inconsistance identitaire.

\section{Objectif de l'étude}

Les auteurs ont alors repris l'analyse de protocoles de Rorschach de patients ayant un trouble de personnalité évitante, afin de :

- mettre rétrospectivement en évidence la prévalence, chez les personnalités évitantes, des traits narcissiques au test de Rorschach ;

- discuter leur signification à la lumière du processus psychothérapique.

\section{Méthodologie}

\subsection{Population}

Le matériel de recherche est constitué de 20 protocoles de Rorschach de patients diagnostiqués, selon les critères DSM-IV-TR, par le psychiatre traitant, comme porteurs d'un trouble évitant de la personnalité, sans troubles affectifs actuels associés.

Ce groupe comprend 11 femmes et 9 hommes, caucasiens, célibataires, d'âge moyen $25,4 \pm 7,8$ ans, majoritairement sans emploi ou avec un emploi précaire ou temporaire.

Les 20 protocoles de Rorschach ont été cotés deux fois : (1) préalablement au début de la psychothérapie et (2) à l'inclusion dans le matériel de recherche, par les deux auteurs. Pour les principales variables d'intérêts de l'étude, le taux de concordance de cotation était de $100 \%$ (pour les réponses reflets, les réponses d'instropection FD). Pour les autres, les pourcentages d'accord par segment étaient dans les standards attendus [15] après quelques ajustements au niveau de la cotation des déterminants et des contenus.

Les cliniciens ayant analysé les données sont tous deux chercheurs et psychologues expérimentés, pratiquant l'examen psychologique et la psychothérapie depuis plus de 15 ans.

L'outil d'investigation est le test projectif de Rorschach (Système Intégré). Cet instrument figure parmi les tests projectifs les plus utilisés au monde $[4,9,25,26]$ et présente de solides propriétés psychométriques [18,23,24,38]. Son utilisation a démontré son intérêt et son efficience dans différents domaines de la psychologie [16].

\subsection{Analyse des données}

Les statistiques descriptives, les comparaisons de moyennes (test de Mann Whtiney), de fréquences (test exact de Fisher) ont été effectuées à l'aide du logiciel Jamovi.

\footnotetext{
1 Variables Rorschach : CDI positif ; Lambda $>\mathrm{N}$.

2 Présence de la variable Reflets $(\mathrm{Fr}+\mathrm{rF})>\mathrm{N}$.
}

\subsection{Déroulement de l'étude}

Pour la recherche de prévalence des caractéristiques évitantes associées aux traits narcissiques, les 20 protocoles de Rorschach ont tous été administrés préalablement à la psychothérapie. Les patients ont donné leur consentement éclairé pour l'utilisation anonyme de leurs réponses à des fins pédagogiques et de recherche.

Les 20 protocoles ont été recueillis durant deux ans. À l'issue de l'examen psychologique, les patients ont commencé leur psychothérapie avec la première auteure. De type intégratif, elle s'est déroulée régulièrement à raison d'une demi-heure par semaine.

Pour la signification des données Rorschach à la lumière du processus psychothérapique, le groupe total a été subdivisé en deux sous-groupes, en fonction de leur adhésion au traitement psychothérapique.

L'adhésion au traitement psychothérapique a été opérationnalisée comme la poursuite, d'un commun accord thérapeute/ patient, de la thérapie, au-delà de la simple amélioration symptomatique.

La non-adhésion au traitement psychothérapique a été opérationnalisée comme le souhait explicite du patient, oral ou écrit, d'interrompre la thérapie, malgré les recommandations du psychothérapeute.

Ainsi, les 20 patients ont été répartis en deux sous-groupes : (1) patients adhérents au traitement psychothérapique (AT), et (2) patients non adhérents au traitement psychothérapique (NAT).

1. Le groupe AT : $n=11$ ( $7 \mathrm{~F}$ et $4 \mathrm{H}$; âge $18-46 ; \mathrm{m}=24,5 \pm 8,7$ ).

2. Le groupe NAT : $n=9$ ( $4 \mathrm{~F}$ et $5 \mathrm{H}$; âge $20-34 ; \mathrm{m}=26,4 \pm 7$ ).

Les deux groupes ne sont pas différents au niveau de l'âge (test de Mann-Withney, $p=0,38$, ns), ni du sexe (test exact de Fisher, $p=0,65, \mathrm{~ns})$.

\section{Résultats}

\subsection{Principaux résultats sur l'ensemble du groupe de patients porteurs de personnalités évitante (TEP)}

Une première partie des résultats est attendue car celle-ci décrit la sémiologie des troubles évitants de personnalité : retrait social, faibles ressources psychiques, peur du rapproché émotionnel, faible estime de soi et stratégie envahissante d'évitement (Tableau 1).

Une deuxième partie des résultats rend compte des difficultés habituellement rencontrées dans une population consultante souffrant de divers troubles de la personnalité et sont systématisés dans le tableau IV.1.2. en fonction de leur signification psychologique et des leurs conséquences sur le processus thérapeutique. Les variables Rorschach indiquent, chez ces patients, la présence de stress ou d'anxiété chronique ainsi que des sentiments d'injustice [7]. La rigidité et les difficultés d'introspection maintiennent les troubles et représentent des formes de résistance à la psychothérapie [5 p. 122]. Cependant, un résultat est inattendu dans une population consultant pour des troubles évitants de la personnalité. Il s'agit de la présence quantitativement importante de traits narcissiques (près de la moitié de notre échantillon).

La présence de traits narcissiques chez des personnalités fonctionnant sur un mode évitant est conceptualisée par certains chercheurs comme une stratégie de coping axée sur l'émotion et visant à restaurer l'estime de soi [10,11]. Grâce à ces stratégies, restauratives sur le plan émotionnel, les personnalités évitantes élaborent des scénarios grandioses au cours desquels elles prennent une revanche sur l'environnement en renversant les rôles et en passant du rôle de dominé à celui de dominant, du rôle d'esclave à celui de tyran. Ces scénarios se mettent en place, à 
Tableau 1

Critères diagnostiques et variables Rorschach pour le groupe TPE.

\begin{tabular}{|c|c|c|}
\hline Critères DSM-IV-TR & Variables Rorschach & Sujets concernés, $n(\%)$ \\
\hline Est réservé dans les relations intimes par crainte d'être exposé à la honte ou au ridicule & $\mathrm{T}=0$ & $18(90)$ \\
\hline Est inhibé dans les situations intrerpersonnelles à cause d'une sentiment de ne pas être à la hauteur & $\mathrm{CDI}>4$ & $15(75)$ \\
\hline Se perçoit comme socialement incompétent, sans attrait ou inférieur & Ego $<\mathrm{N}$ & $15(75)$ \\
\hline \multirow[t]{2}{*}{ Évite les activités sociales qui impliquent des contacts importants avec autrui } & $\mathrm{CDI}>4$ & $15(75)$ \\
\hline & Ego $<\mathrm{N}$ & $15(75)$ \\
\hline Évite la complexité de la vie quotidienne & $\mathrm{L}>99$ & $10(50)$ \\
\hline
\end{tabular}

Tableau 2

Variables Rorschach additionnelles - Troubles de la personnalité.

\begin{tabular}{|c|c|c|c|}
\hline$n \%$ & Variable Rorschach & Signification psychologique & Conséquences sur le processus psychothérapique \\
\hline 90 & $\mathrm{FD}=0$ & Difficultés d'introspection & $\begin{array}{l}\text { Absence d'objectivité dans les relations intra et interpersonnelles. } \\
\text { Remise en question difficile }\end{array}$ \\
\hline 45 & Reflet $>0$ & $\begin{array}{l}\text { Traits narcissiques-surestimation de la valeur personnelle : } \\
\text { déni, idéalisation et externalisation }\end{array}$ & $\begin{array}{l}\text { Mécanismes qui peuvent s'opposer au changement psychique par } \\
\text { attribution externe }\end{array}$ \\
\hline 40 & Ebper et $\mathrm{a} / \mathrm{p}>\mathrm{N}$ & Rigidité des croyances et des points de vue & $\begin{array}{l}\text { Maintien et renforcement des troubles par résistance au } \\
\text { changement }\end{array}$ \\
\hline 40 & $\mathrm{D}<0 ; \operatorname{Ajd}<0$ & Surcharge en stress situationnel et/ou chronique & Atout pour la thérapie, motivation à changer pour moins souffrir \\
\hline 35 & $\mathrm{~S}>\mathrm{N}$ et $\mathrm{AG}>\mathrm{N}$ & $\begin{array}{l}\text { Ressentis d'opposition et de d'injustice et agressivité dans } \\
\text { les relations interpersonnelles }\end{array}$ & $\begin{array}{l}\text { Résistance au changement par des difficultés dans le maintien de } \\
\text { d'alliance }\end{array}$ \\
\hline
\end{tabular}

certains moments, lorsqu'elles sont conscientes d'avoir réussi dans un des domaines de leur vie et les protègent de la confrontation à une image négative de soi [10].

À la lumière de ces constatations, nous avons tenté de dégager leur signification psychologique et leur impact sur le processus thérapeutique. En effet, lorsqu'on place ces résultats dans le cadre du processus psychothérapique, on observe que 9 des 20 patients TEP de notre groupe ont terminé prématurément le suivi, malgré les recommandations et les encouragements de leur thérapeute à poursuivre. Les raisons invoquées ont été principalement :

- pour les 9, le sentiment d'amélioration significative de l'état psychologique par rapport au début du traitement ;

- pour 8 des 9 patients, la présence d'un nouvel équilibre extérieur concrétisé dans de nouvelles opportunités de travail, des relations amoureuses satisfaisantes ou une affiliation communautaire.

Or, même si l'on constate que la non-adhérence complète au traitement psychothérapique est une donnée fréquemment citée dans la littérature indiquant que :

- 20 à $50 \%$ des patients terminent prématurément le traitement psychothérapique ;

- le modèle dose/effet montre la présence d'une amélioration psychologique significative au terme de 15 à 20 séances pour $50 \%$ des patients en psychothérapie [22].

On s'interroge sur le processus qui produit cet effet en analysant les caractéristiques psychologiques des patients du groupe NAT par comparaison au groupe AT. Les variables Rorschach correspondant à leur fonctionnement psychique sont systématisées dans le Tableau 2.

4.2. Comparaison des profils Rorschach des sous-groupe de patients NAT et $A T$

Au test exact de Fischer, ces patients se différencient de manière significative, par rapport à la présence des variables suivantes : stress et anxiété chronique, traits narcissiques et rigidité plus fréquents chez les NAT (Tableau 3).
Tableau 3

Comparaison des scores des patients AT et NAT.

\begin{tabular}{llll}
\hline Variables Rorschach & NAT $(n=9), \%$ & AT $(n=11)$ & Test exact de Fisher, $p$ \\
\hline Reflet $>\mathrm{N}^{\mathrm{a}}$ & $9-100$ & 0 & $<0,01$ \\
D et Dajd $<\mathrm{N}^{\mathrm{b}}$ & $8-90$ & 0 & $<0,01$ \\
EBper et a/p $\mathrm{p}^{\mathrm{c}}>\mathrm{N}$ & $8-90$ & 0 & $<0,01$ \\
\hline a Traits narcissiques. & & \\
b Stress chronique. & & \\
c Rigidité. &
\end{tabular}

On constate ainsi que les patients TPE de ce groupe présentent :

- une importante détresse psychologique ;

- des mécanismes de défenses de la série immature [2, p. 53-55], de type idéalisation de soi, déni, externalisation qui entretiennent une grandiosité de l'image de soi ;

- une rigidité cognitive très présente.

\section{Discussion}

Comment conceptualiser ces données et quel rôle jouent-elles dans le processus thérapeutique et plus particulièrement dans l'adhésion au traitement? Nous formulons l'hypothèse suivante: la souffrance psychique, ainsi que le sentiment de vulnérabilité, représentent des facteurs motivationnels incitant les patients à débuter le traitement psychologique.

au moment où une amélioration affective est obtenue, ses patients pourraient se situer à un moment crucial de leur travail psychothérapique. Du fait de la présence de faibles capacités introspectives, des mécanismes de défense qui les renforcent et de la rigidité des croyances. En effet, au moment où la thérapie est susceptible d'aborder des niveaux psychologiques plus intimes, les patients TEP pourraient réactiver le processus d'anticipation anxieuse et redouter, s'ils allaient plus loin dans le travail thérapeutique, le retour d'une importante souffrance du fait de la honte qui découle de la confrontation avec un soi imparfait et indigne [10]. En conséquence, la peur d'un débordement émotionnel futur peut devenir envahissante et imposer le retour à des stratégies de coping anciennes et rassurantes, de type évitement et 
compensation narcissique, qui leur donnent l'illusion de maintenir à distance la détresse psychique [10].

Dans cette perspective, l'interruption prématurée de la psychothérapie chez près de la moitié des patients TEP de notre échantillon pourrait s'analyser comme la conséquence d'un conflit approche-évitement où l'évitement devient un style de fonctionnement/résolution, équilibré par des facteurs externes. Il s'agirait donc d'un conflit entre le défi thérapeutique du changement (approche) et la menace de souffrance (évitement) dans sa poursuite.

Dans ce conflit, l'approche se manifeste aux premiers stades de la psychothérapie, par la motivation à chercher de l'aide pour stopper la détresse psychologique et trouver de la sécurité dans l'alliance thérapeutique.

L'évitement s'installe lorsque le soulagement de la détresse devient effectif. Le sentiment de vulnérabilité reste encore présent et alimente, si la thérapie continue, la peur d'une plus grande souffrance induite par le dévoilement de soi dans ses imperfections. Le processus de changement est ainsi bloqué par les " les traits narcissiques " et par l'idéalisation de soi contenue dans les scénarios de grandeur qui interviendraient avec "la réussite " d'un mieux-être psychologique. Dans ce cas, le fonctionnement du sujet se réorganise, à nouveau, sur un mode évitant plus supportable.

Ou encore, ce dévoilement de soi pourrait peut-être aller jusqu'au fantasme redouté que, voyant son " vrai visage ", le thérapeute finisse par dysqualifier le patient et souhaite alors rompre le lien, interrompre la thérapie, au risque de lui faire subir un abandon si redouté, une blessure narcissique insupportable. À l'instar du modèle de Young (2017) [42], une stratégie de contreattaque du schéma d'abandon leur permettrait de "quitter " pour " ne pas être quitté " et ne pas perdre la face.

Ainsi, si l'évitement " gagne la bataille psychothérapique » c'est parce que :

- à la lumière du modèle de la psychologie de la santé : la perception des risques encourus (souffrance psychique) est supérieure à celle des bénéfices anticipés [35]. Dans notre étude, l'expérience de la tension interne, du stress et le sentiment de vulnérabilité pourraient constituer une expérience psychologiquement éprouvante pour ces patients qui deviennent réticents à l'idée de discuter ouvertement et au long cours de leurs problématiques intimes, par peur de souffrir à nouveau intensément, notamment de l'abandon. Le coût psychologique d'un changement en profondeur est donc perçu comme trop élevé et diminue la tolérance au processus psychothérapique ;

- à la lumière de la théorie de l'autodétermination [31], certains patients diffèrent dans la manière de définir leur sentiment de compétence, dans une tâche donnée. La définition personnelle détermine le degré de leur engagement et d'endurance. Dans notre étude, la difficulté qu'ont ces patients NAT à réfléchir constructivement sur eux-mêmes peut être considérée comme une croyance dysfonctionnelle rigide les convainquant de leur incapacité de réussir dans une tâche telle que l'introspection et le processus de changement ;

- à la lumière des conceptions sur l'adhésion au traitement, la présence de traits narcissiques peut être envisagée comme un facteur freinateur qui entretient, par les mécanismes de défense spécifiques, la résistance au changement [6]. Ce qui permet aux patients évitants d'éviter d'approfondir leurs problématiques ou de faire face authentiquement à leurs ressentis de honte ou de culpabilité. Le refuge dans des scénarios grandioses devient une forme d'évitement cognitif où la compensation narcissique permet de ne pas évoquer des situations menaçantes ou des images négatives de soi [1, p. 80].

\section{Limites de l'étude}

Si cette étude a apporté un point de vue clinique sur la prise en charge des sujets porteurs de troubles évitants de personnalité, elle comporte certaines limites. En premier lieu, il s'agit d'une étude rétrospective, sur un petit échantillon qui, malgré son intérêt dans "l'enrichissement » de la recherche par l'observation clinique [5,36, p. 484] ne permet pas l'élaboration d'un protocole contrôlé et le retest à l'interruption de la psychothérapie n'a pas pu être réalisé.

\section{En conclusion}

Certains patients porteurs de troubles évitants de la personnalité vivent le processus psychothérapique comme un terrible conflit de type approche-évitement. La rigidité, la présence de traits narcissiques et l'anxiété chronique peuvent être considérées comme des facteurs internes aux patients qui s'opposent à la poursuite du processus psychothérapique. Pour certains patients TPE, il existe un degré de " tolérabilité » de la psychothérapie, qu'ils ne peuvent dépasser par peur d'une trop forte souffrance psychique.

Mais comment expliquer que certains patients ayant une personnalité évitante présentent des traits narcissiques et d'autre pas ? Voici une autre question de recherche qui pourrait impliquer la présence plus marquée d'un schéma d'abandon ou de stratégies adaptatives particulières trouvées par ces patients (de type contreattaque) [42]. Mais il faudrait, pour tester cela, pouvoir approfondir leur anamnèse, rechercher leur éventuels traumatismes ou manques, sans qu'ils s'en sentent fragilisés. .

En corollaire, et dans une autre perspective, une autre interrogation émerge : comment définir une psychothérapie réussie ? La question se pose du point de vue du patient et de celui du psychothérapeute, sachant qu'ils ne seront pas nécessairement convergents.

\section{Déclaration de liens d'intérêts}

Les auteurs déclarent ne pas avoir de liens d'intérêts.

\section{Références}

[1] Borkovec TD, Alcaine OM, Behar E. Avoidance, theory of worry and generalized anxiety disorder. In: Generalized anxiety disorder: advances in research and practice. New York, NY, US: Guilford Press; 2004. p. 77-108.

[2] Callahan S, Chabrol H. Mécanismes de défense et de coping. Paris: Dunod; 2013. p. $51-90$

[3] Carrigan MH, Randall CL. Self-medication in social phobia: a review of the alcohol literature. Addict Behav 2003;28:269-84.

[4] Castro D, Meljac C, Joubert B. Pratiques et outils des psychologues cliniciens français. Les enseignements d'une enquête. Prat Psychol 1996;4:73-80.

[5] Castro D. Pratique de l'examen psychologique en clinique adulte. WAIS IV, RORSCHACH, TAT, MMPI-2. Paris: Dunod; 2016.

[6] Castro D, Zoute C, Le Rohellec J. Adhésion au traitement prescrit et traits de personnalité dans une population de patients atteints de schizophrénie. Ann Med Psychol 2004;162:262-70. http://dx.doi.org/10.1016/j.amp.2003.06.004.

[7] Charnas JW, Hilsenroth MJ, Zodan J, Blais MA. Should I stay or should I go? Personality Assessment Inventory and Rorschach indices of early withdrawal from psychotherapy. Psychotherapy 2010;47:484-99. http://dx.doi.org/ 10.1037/a0021180.

[8] Debray Q Nollet D. Les personnalités pathologiques. Approche cognitive et thérapeutique. Paris: Masson; 2011.

[9] De Rinquesen V. Pratiques et outils des psychologues cliniciens français : étude comparative entre 1996 et 2011. Etudes Prat Psychol 2013;1:66-77.

[10] Dimaggio G, Procacci M, Nicolò G, Popolo R, Semerari A, Carcione A, et al. Poor metacognition in Narcissistic and Avoidant Personality Disorders: four psychotherapy patients analysed using the Metacognition Assessment Scale. Clin Psychol Psychother 2007;14:386-401. http://dx.doi.org/10.1002/cpp.541.

[11] Dimaggio G, Semerari A, Carcione A, Procacci M, Nicolò G. Toward a model of self-pathology underlying personality disorders: narratives, metacognition, interpersonal cycles and decision-making processes. J Pers Disord 2006;20:597-617. http://dx.doi.org/10.1521/pedi.2006.20.6.597.

[12] Dimaggio G, Popolo R, Salvatore G. Chapter 8 - Formulation of functioning for avoidant personality disorder in metacognitive interpersonal therapy. In: Kramer U, editor. Case formulation for personality disorders [Internet]. 
demic Press; 2019 [cité 5 mars 2019 ; 137 ; 60 ; disponible sur : http://www. sciencedirect.com/science/article/pii/B9780128135211000084].

[13] Eikenaes I, Hummelen B, Abrahamsen G, Andrea H, Wilberg T. Personality functioning in patients with avoidant personality disorder and social phobia. J Pers Disord 2013;27:746-63. http://dx.doi.org/10.1521/pedi_2013_27_109.

[14] Eikenæs I, Pedersen G, Wilberg T. Attachment styles in patients with avoidant personality disorder compared with social phobia. Psychol Psychother 2016;89(3):245-60. http://dx.doi.org/10.1111/papt.12075.

[15] Exner J. Issues and methods in Rorschach research. Mahwah: Lawrence Erlbaum Associates; 1995.

[16] Frandsen FW, Simonsen S, Poulsen S, Sørensen P, Lau ME. Social anxiety disorder and avoidant personality disorder from an interpersonal perspective. Psychol Psychother 2019. http://dx.doi.org/10.1111/papt.12214.

[17] Fouques D. Évaluation des psychothérapies : l'apport du Rorschach (S.I.). Psychol Fr 2004;49:25-32. http://dx.doi.org/10.3917/pcp.021.0195.

[18] Havranek MM, Volkart F, Bolliger B, Roos S, Buschner M, Mansour R, et al. The fear of being laughed at as additional diagnostic criterion in social anxiety disorder and avoidant personality disorder? PLOS ONE 2017;12:e0188024. http://dx.doi.org/10.1371/journal.pone.0188024.

[19] Hilsenroth MJ, Stricker G. A consideration of challenges to psychological assessment instruments used in forensic settings: Rorschach as exemplar. J Pers Assess 2004;83:141-52. http://dx.doi.org/10.1207/s15327752jpa8302_08.

[20] Hilsenroth MJ, Cromer TD. Clinician interventions related to alliance during the initial interview and psychological assessment. Psychotherapy 2007;44:205-18. http://dx.doi.org/10.1037/0033-3204.44.2.20.

[21] Hilsenroth MJ, Peters EJ, Ackerman SJ. The development of therapeutic alliance during psychological assessment: patient and therapist perspectives across treatment. J Pers Assess 2004;83:332-44. http://dx.doi.org/10.1207/ s15327752jpa8303_14.

[22] Jourdy R, Petot J-M. La théorie des cing facteurs. Profils de personnalité selon la théorie des cinq facteurs et troubles de la personnalité chez des patients déprimés. An Med Psychol 2018;176:219-24 [10.1016 \%2Fj.amp.2017.01.024].

[23] Lambert MJ. Outcome in psychotherapy: the past and important advances. Psychotherapy 2013;50:42-51. http://dx.doi.org/10.1037/a0030682.

[24] Meyer GJ, Finn SE, Eyde LD, Kay GG, Moreland KL, Dies RR, et al. Psychological testing and psychological assessment: a review of evidence and issues. Am Psychol 2001;56:128-65. http://dx.doi.org/10.1037//0003-066X.56.2.128.

[25] Meyer GJ. The reliability and validity of the Rorschach and Thematic Apperception Test (TAT) compared to other psychological and medical procedures: an analysis of systematically gathered evidence. In: Comprehensive handbook of psychological assessment: Vol 2: personality assessment. Hoboken, NJ, US: John Wiley \& Sons Inc.; 2004. p. 315-42.

[26] Norcross JC, Hedges M, Castle PH. Psychologists conducting psychotherapy in 2001: a study of the Division 29 membership. Psychotherapy 2002:39:97-102.

[27] Norcross JC, Rogan JD. Psychologists conducting Psychotherapy in 2012: current practices and historical trends among Division 29 members. Psychotherapy 2013;50:490-5. http://dx.doi.org/10.1037/a0033512.
[28] Pellecchia G, Moroni F, Colle L, Semerari A, Carcione A, Fera T, et al. Avoidant personality disorder and social phobia: does mindreading make the difference? Compr Psychiatry 2018;80:163-9. http://dx.doi.org/10.1016/ j.comppsych.2017.09.011.

[29] Porcerelli JH, Dauphin VB, Ablon JS, Leitman S, Bambery M. Psychoanalysis with avoidant personality disorder: a systematic case study. Psychotherapy 2007;44:1-13. http://dx.doi.org/10.1037/0033-3204.44.1.1.

[30] Presniak MD, Olson TR, Porcerelli JH, Dauphin VB. Changes in defensive functioning in a case of avoidant personality disorder. Psychotherapy 2010;47:134-9. http://dx.doi.org/10.1037/a0018838.

[31] Rees CS, Pritchard R. Brief cognitive therapy for avoidant personality disorder. Psychotherapy 2015;52:45-55. http://dx.doi.org/10.1037/a0035158.

[32] Reeve J, Deci E, Ryan R. Self-determination theory: a dialectical framework for understanding sociocultural influences on student motivation; 2004 [31 p.]

[33] Sanislow CA, Bartolini E, Zoloth. Avoidant personality disorder. In: Ramachandran VS, editor. Encyclopedia of human behavior. 2nd Ed., San Diego: Academic Press; 2012. p. 257-66

[34] Skodol AE, Bender DS, Oldham JM, Clark LA, Morey LC, Verheul R, et al Proposed changes in personality and personality disorder assessment and diagnosis for DSM-5 Part II: clinical application. Pers Disord 2011;2:23-40 [10.10372Fa0021892].

[35] Sørensen KD, Råbu M, Wilberg T, Berthelsen E. Struggling to be a person: lived experience of avoidant personality disorder: SØRENSEN, et al.. J Clin Psychol [Internet] 2019;75:664-6 [cité 5 mars 2019 ; disponible sur : http://doi.wiley.com/10.1002/jclp.22740].

[36] Swift JK, Greenberg RP, Tompkins KA, Parkin SR. Treatment refusal and premature termination in psychotherapy, pharmacotherapy, and their combination: a meta-analysis of head-to-head comparisons. Psychotherapy 2017;54:47-57. http://dx.doi.org/10.1037/pst0000104.

[37] Stiles WB. Theory building enriching and fact gathering: alternative purposes of psychotherapy research. In: Gelo OCG, Pritz A, Rieken B, editors. Psychotherapy research: foundations, process, and outcome. New York, NY, US Springer-Verlag Publishing; 2015. p. 159-79. http://dx.doi.org/10.1007/9783-7091-1382-0 8

[38] Torgersen S, Kringlen E, Cramer V. The prevalence of personality disorders in a community sample. Arch Gen Psychiatry 2001;58:590.

[39] Viglione DJ, Hilsenroth MJ. The Rorschach: facts, fictions, and future. Psychol Assess 2001;13:452-71.

[40] Wright AGC, Pincus AL, Lenzenweger MF. Development of personality and the remission and onset of personality pathology. J Pers Soc Psychol 2011;101:1351-8. http://dx.doi.org/10.1037/a0025557.

[41] Weinbrecht A, Schulze L, Boettcher J, Renneberg B. Avoidant personality disorder: a current review. Curr Psychiatry Rep 2016;18:29. http:/ dx.doi.org/10.1007/s11920-016-0665-6.

[42] Young JE, Klosko JS, Weishaar ME. Schema therapy: a practitioner's guide. New York: Guilford Press; 2003. 